\title{
Material flow in Friction Stir Welding
}

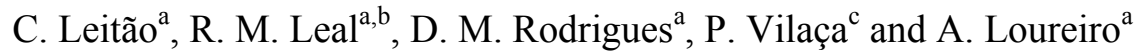 \\ ${ }^{\text {a }}$ CEMUC - Department of Mechanical Engineering, University of Coimbra, Portugal \\ ${ }^{\mathrm{b}}$ ESAD.CR-IPL - Polytechnic Institute of Leiria, Caldas da Rainha, Portugal \\ ${ }^{c}$ DEM-IST, Technical University of Lisbon, Portugal \\ carlos.leitao@dem.uc.pt
}

Friction stir welding (FSW) is a solid-state joining technique initially developed for aluminium alloys. The heat generated by a rotating tool softens the material in the vicinity of the tool. The material undergoes intense plastic deformation following quite complex paths around the tool, depending on the tool geometry, process parameters and material to be welded $[1,2]$. The comprehension of the material flow is essential to prevent voids and other internal defects which may form during welding [3]. Several techniques have been used for tracking material flow during FSW such as metallography, the use of a marker material as a tracer or the flow visualization by FSW of dissimilar materials or even the X-ray and computer tomography $[4,5]$. Some of these techniques are useless in the analysis of welds in homogenous materials or welds between materials of the same group. The aim of this investigation is tracking the material flow in FSW between $1 \mathrm{~mm}$ thick sheets in aluminium alloys AA 5182-H111 and AA 6016-T4, currently used in automotive industry.

Welds were performed in a conventional milling machine, in tool control position, using a tool composed by a shoulder of $10 \mathrm{~mm}$ in diameter with an $8^{\circ}$ conical cavity and a left threaded cylindrical probe of $3 \mathrm{~mm}$ in diameter and $0.9 \mathrm{~mm}$ in length, see Fig 1. A tool rotation speed of 1800 $\mathrm{rpm}$, a travel speed of $160 \mathrm{~mm} / \mathrm{min}$ and a tool tilt angle of $2.5^{\circ}$ were used in the welds.

A convenient metallographic technique was used to reveal the material flow path, as is illustrated in Fig. 2 and Fig. 3. These figures display respectively horizontal (at half thickness) and transversal cross sections of a weld. Cross sections were polished and etched with modified Poulton's reagent in order to get the differential etching shown in the figure Fig. 3a). Aluminium alloy 5182-H111, located in the advancing side of the weld, shows a convenient etching, revealing the grain boundaries of the parent material contrarily to AA 6016-T4 that displays a weak contrast of the structure. Fig. 2 shows in the central zone of the weld, currently denominated thermomechanically affected zone, the deposition of intercalated layers of two different materials spaced each other of approximately $0.09 \mathrm{~mm}$. This spacing corresponds to the tool advance per revolution and suggests that booth materials are drawn around the tool from the leading to the trailing side of the tool and deposited in the rear in a cyclic mode. It is not clear at this stage if there is a real mixture of both materials, because they only show different morphology. Onion ring patterns can be observed in transversal cross section of the welds, Fig. 3, in the central zone usually designated as nugget. These patterns result of the interaction between the material flow driven by the rotating pin and the shoulder driven flow. This interaction is clearly shown by the deformation of the onion rings in the advancing side of the weld, where a small expulsion of material (flash) is observed. Several explanations have been given for the formation of these patterns. Some authors state the onion ring patterns arise from the geometric nature of stacking of layers in the pin driven material [6] or from 
the difference in the degree of deformation within layers deposited by the tool. Etching contrast can also arise from local chemical composition change in transferred layers, as already observed for a precipitation hardenable aluminium alloy [7].

Another metallographic technique was used in order to elucidate the origin of the onion ring patterns. A reagent named R2, composed of methanol $(25 \mathrm{ml})$, hydrochloric acid $(25 \mathrm{ml})$, nitric acid $(25 \mathrm{ml})$ and a drop of hydrofluoric acid, swabbed on the specimen's surface at room temperature for $20 \mathrm{~s}$ was used to expose precipitates. The concentration of precipitates in deposited layers can trace the materials flow path as well the distribution of plasticized materials in the weld. Fig. $3 b$ ) shows that this reagent enhances mainly the precipitates of aluminium alloy 5182-H111. The distribution of precipitates in the nugget is quite similar to the distribution of onion rings displayed by etching with Poulton's reagent, showing also an onion ring structure. This suggests the onion ring structure is composed of intercalated layers of AA 5182-H111, those with precipitates, and AA 6016-T4, those without visible precipitates.

The X-Ray elemental map distribution of specific elements of each material was performed in order to validate the observations done using metallographic technique mentioned above. The contents in magnesium and silicon in different zones of the welds were investigated because alloy 5182-H111 is rich in magnesium (\%wt $-\mathrm{Mg}-4-5$ and $\mathrm{Si}<0.2)$ and alloy 6016-T4 is rich in silicon $(\% \mathrm{wt}-\mathrm{Mg}-$ 0.25-0.6 and $\mathrm{Si}-1-1.5)$. Fig. 4a) illustrates the distribution of $\mathrm{Mg}$ in the nugget close the retreating side. Regions enriched in $\mathrm{Mg}$ are enhanced by the blue bands, corresponding to zones constituted mainly of AA 5182-H111, as suggested above by the metallographic technique. Regions enriched in $\mathrm{Si}$ are revealed by light-coloured dots in Fig. 4b). The concentration of these light-coloured dots is essentially outside the dark bands rich in $\mathrm{Mg}$, but there are also some dots inside the bands giving the idea of some mixture of materials in the layers.

In conclusion, results presented above show that the pin driven flow is predominant in determining the characteristics of the nugget of these welds, yielding an onion ring structure caused by the flux of layers of plasticized material around the pin. Metallographic techniques can be used with success in revealing material flow paths in heterogeneous friction stir welds.

Acknowledgements

The authors are indebted to the Portuguese Foundation for the Science and Technology (FCT) and FEDER for the financial support and to Novelis Switzerland SA for supplying the aluminium sheets.

References

[1] K. Kumar and S. V. Kailas, Materials Science and Engineering: A, In Press.

[2] Z. W. Chen and S. Cui, Scripta Materialia 58 (2008) 417-420.

[3] R. M. Leal and A. Loureiro, Materials \& Design 29 (2008) 982-991.

[4] A. P. Reynolds, Scripta Materialia 58 (2008) 338-342.

[5] H. N. B. Schmidt, T. L. Dickerson, and J. H. Hattel, Acta Materialia 54 (2006) 1199-1209.

[6] K. N. Krishnan, Materials Science and Engineering A 327 (2002) 246-251.

[7] M. A. Sutton et al, Materials Science and Engineering A 323 (2002) 160-166. 


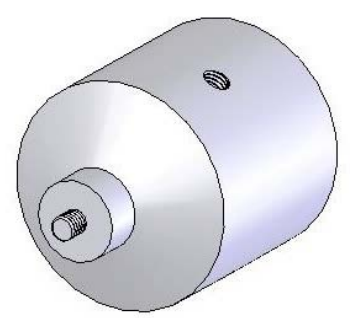

Fig 1 - Friction stir welding tool.

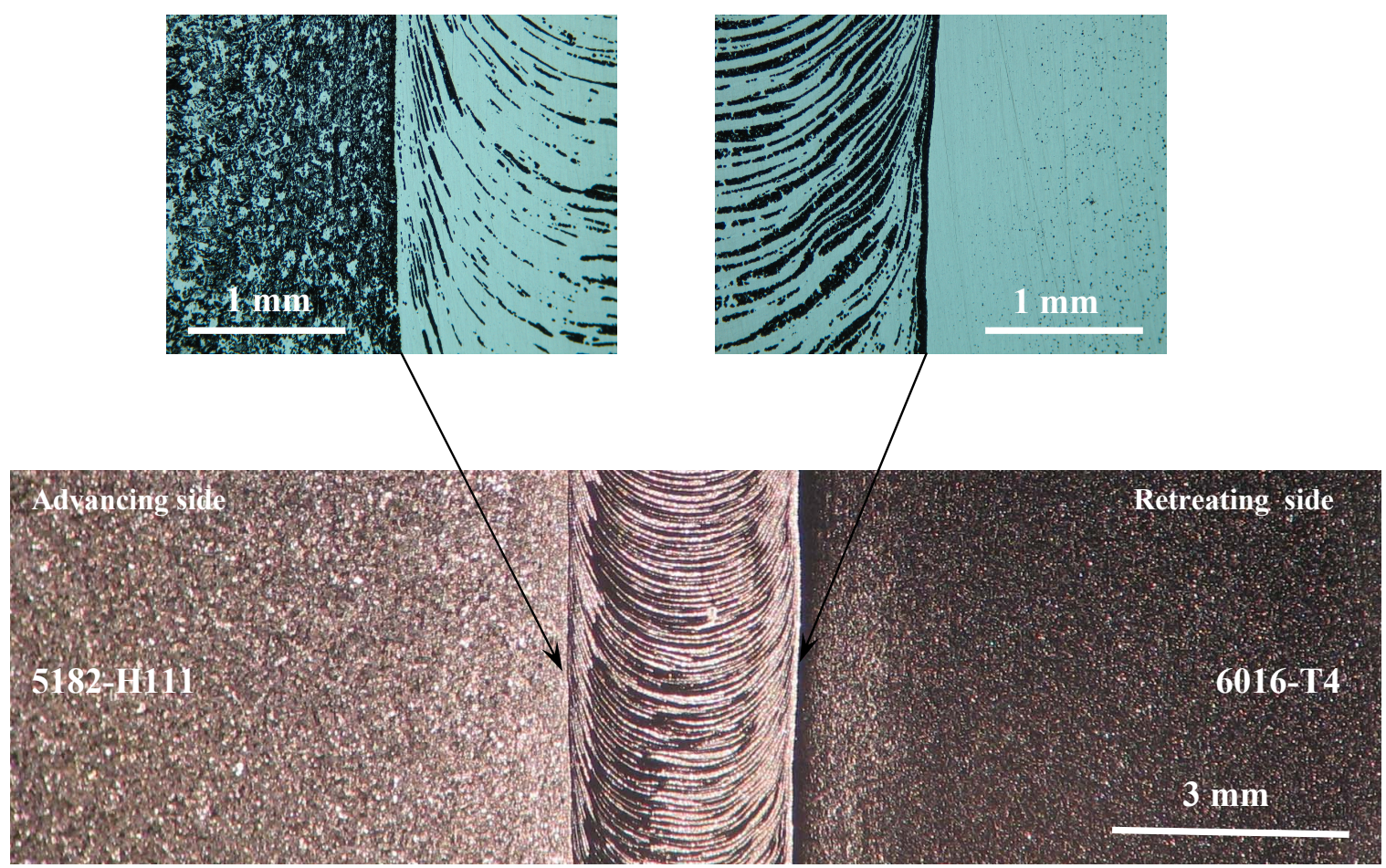

Fig 2 - Horizontal cross section (at half thickness) of a weld (optical microscopy). 

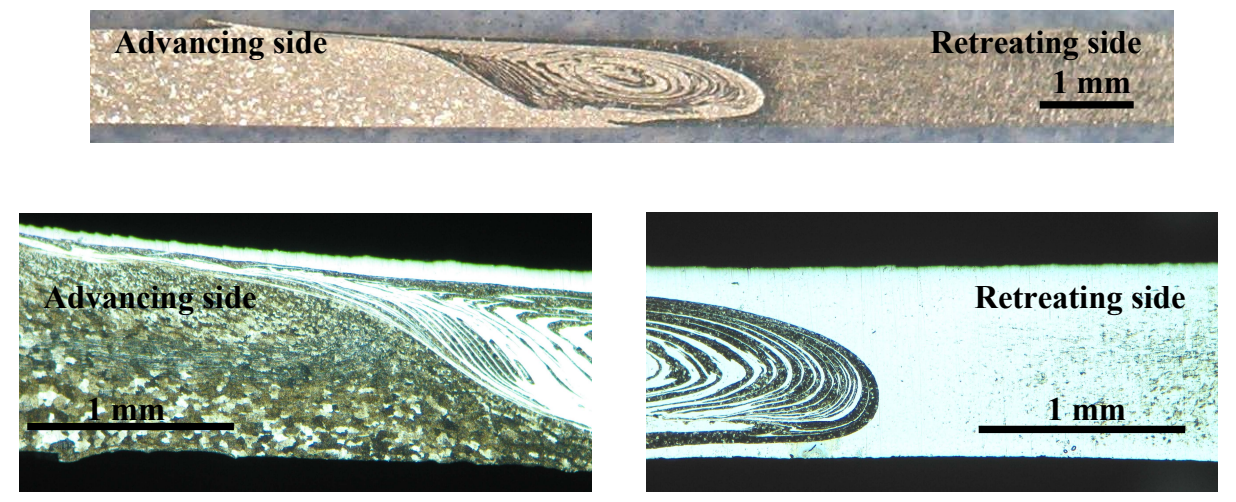

a)

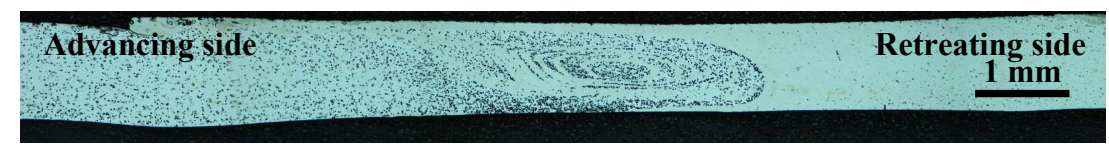

b)

Fig 3 - Transversal cross section of a weld: a) Modified Poulton's reagent; b) R2 reagent (optical microscopy).
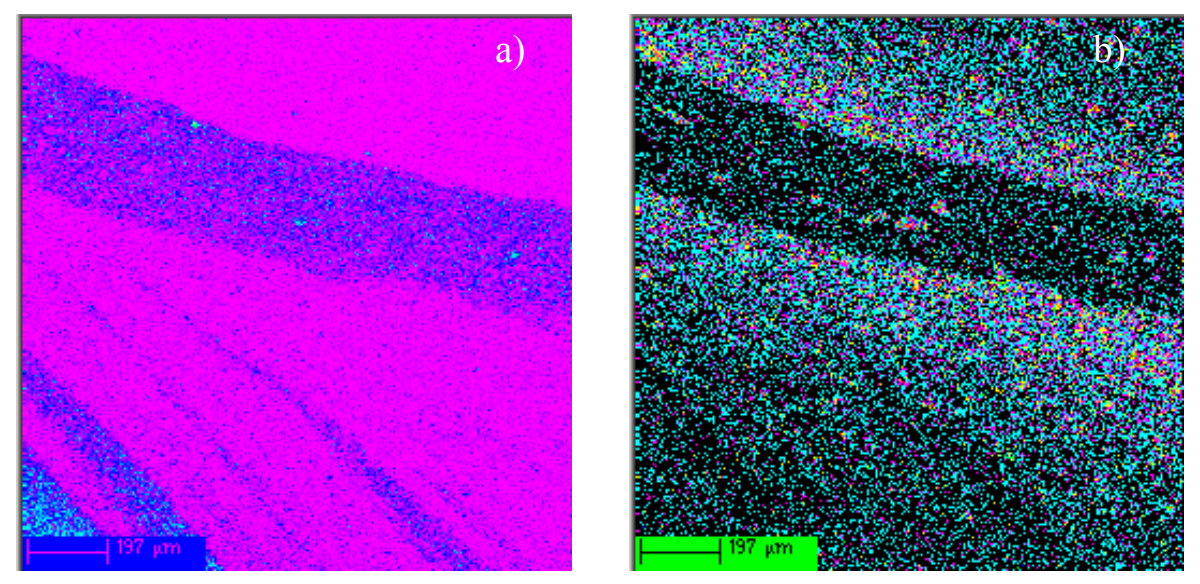

Fig 4 - Elemental map distribution of a) $\mathrm{Mg}$; and b) Si. 